\title{
A novel technique for the measurement of eyelid contour to compare outcomes following Muller's muscle-conjunctival resection and external levator resection surgery
}

\author{
Jennifer Danesh ${ }^{1} \cdot$ Shoaib Ugradar ${ }^{2} \cdot$ Robert Goldberg $^{1,2} \cdot$ Daniel B Rootman $^{1,2,3}$
}

Received: 20 December 2017 / Revised: 5 March 2018 / Accepted: 5 April 2018 / Published online: 24 May 2018

(c) The Royal College of Ophthalmologists 2018

\begin{abstract}
Background Both external levator resection (ELR) and Müller's muscle-conjunctival resection (MMCR) are procedures well known to improve marginal reflex distance (MRD1) in patients with ptosis. This study aims to understand differential post-operative changes in eyelid contour for MMCR and ELR surgery.

Methods In this cross-sectional cohort study, patients affected by involutional ptosis were randomized into two groups: those who underwent ELR or MMCR surgery. Pre-operative and late post-operative photographs were obtained. Digital analysis of the lid contour was performed by measuring the vertical distance from a line intersecting the center of the pupil to the eyelid margin at 10 positions at $2 \mathrm{~mm}$ intervals. Mean distance at each position was compared pre- and post-operatively between the groups.

Results The final sample included 60 eyes from 39 patients, 30 eyes per group. At each time point there was significant variation in height across the eyelid $(p<0.05)$. A significant $(p<0.05)$ group difference in vertical height was noted only at the 2 and $4 \mathrm{~mm}$ temporal positions in the post-operative analysis, with the ELR group being slightly higher. There were no significant differences in MRD1, pre- or post-operatively, between the ELR and MMCR groups.

Conclusions Both ELR and MMCR are effective at elevating the eyelid in multiple positions across the length of the eyelid. Although they do not produce significantly different MRD1 results, ELR was associated with a greater eyelid height at the 2 and $4 \mathrm{~mm}$ temporal positions.
\end{abstract}

\section{Introduction}

Multiple anatomic and physiologic elements including shape, height, contour, and mobility are essential to the cosmetic and functional role of the eyelid [1]. Despite the complexity of this system, eyelid position is frequently described using the

This work was presented at the 2016 Annual Meeting of the Association for Research in Vision and Ophthalmology (ARVO).

Daniel B Rootman

rootman@jsei.ucla.edu

1 David Geffen School of Medicine, University of California, Los Angeles, CA, USA

2 Division of Orbital and Ophthalmic Plastic Surgery, Stein Eye Institute, University of California, Los Angeles, CA, USA

3 Division of Orbital and Ophthalmic Plastic Surgery, Doheny Eye Center, University of California, Los Angeles, CA, USA marginal reflex distance (MRD1) alone [2-6]. While this may describe the central portion of the eyelid, it provides little information regarding abnormalities in eyelid contour such as: notches, peaks, flare, or other deformities.

Such information regarding lid contour would be of value in assessing various pathologic states including thyroid eye disease, lacrimal expansion, and various traumatic conditions. Additionally, and of particular interest to surgeons, are iatrogenic alterations in the eyelid contour after surgery.

Classically, the assessment of surgical change in eyelid contour has been qualitative, typically including descriptors such as "acceptable", "good", or "excellent", although some more formal grading systems have been proposed [7, 8]. Such systems typically lack standardization across raters or centers and are therefore subjective. Standardized quantitative data regarding eyelid contour outcomes would be of significant value in describing the comparative effectiveness of various surgical interventions.

Some groups have proposed using measurements based on Multiple Radial mid pupil Lid Distances (MPLDs). This 
system places a common origin in the center of the pupil, with multiple radial lines extending out from this point to the upper lid margin [9]. A limitation of the MPLD system is that when comparing cases with different eyelid height, the measurements do not correspond to the same positions on the eyelid since the radial lines transect the lid at different points, making a comparison inaccurate.

This study aims to define a standardized and repeatable approach to measuring eyelid contour, and to utilize this technique to assess relative changes in contour for patients alternately undergoing two different surgical procedures for ptosis: external levator resection (ELR) and Müller's muscle-conjunctival resection (MMCR) surgery.

\section{Methods}

In this comparative, cross-sectional cohort study, two populations of subjects were defined. The first underwent Müller's muscle-conjunctival resection without tarsectomy (MMCR) and the second underwent external levator resection (ELR) surgery for the management of eyelid ptosis. This study complied with the policies and principles set forth by the University of California Los Angeles Institutional Review Board and adhered to the tents of the declaration of Helsinki.

\section{Patient selection}

Patients were included if they were over the age of 18 , demonstrated involutional upper eyelid ptosis with levator excursion $>12 \mathrm{~mm}$ and underwent surgical correction. Patients were excluded if they had any history of previous upper eyelid surgery, orbital or eyebrow disease or underwent concurrent upper or lower eyelid procedures (specifically including but not limited to: blepharoplasty, browpexy, and/or lateral canthal tightening). Patients were also excluded if they demonstrated features of any condition that may influence upper eyelid contour such as thyroid orbitopathy, a lacrimal or orbital mass, neuromuscular disease, or facial palsy. Botulinum toxin washout period was a minimum of 5 months prior to study entry.

\section{Image analysis}

Pre-operative and post-operative photographs were obtained parallel to the plane of the face, with the patient in primary position looking directly in to the camera.

Digital image analysis was performed using ImageJ (National Institutes of Health, Bethesda, Maryland). To ensure intra subject standardization of the eyelid to an axis parallel to the horizon, a line was drawn between the patient's lateral canthi and the photographs were rotated to arrange this line parallel to the vertical borders of the digital file. This was

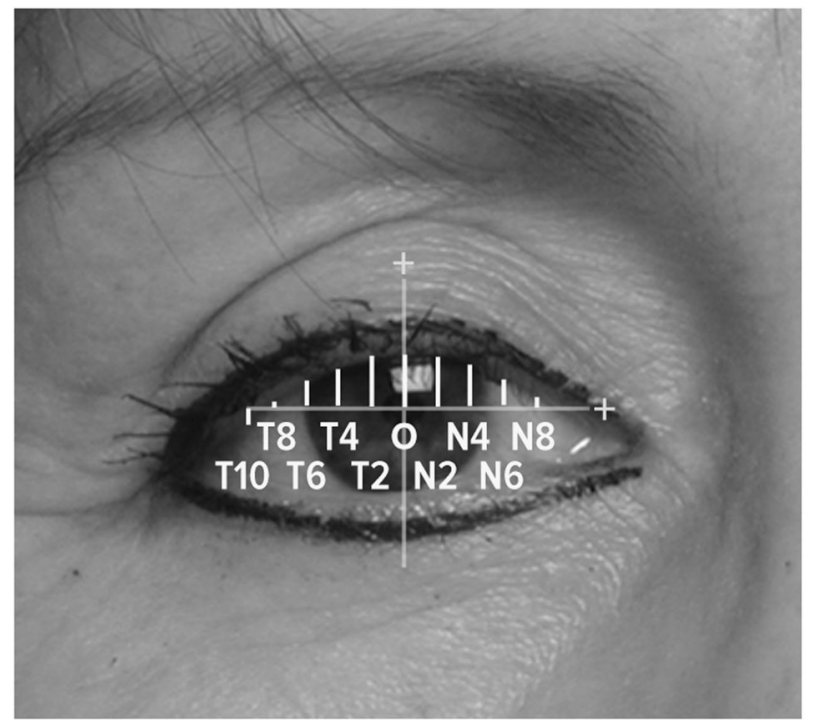

Fig. 1 Measurement positions

performed for each image. Measurements were calibrated using a standard white-to-white diameter of $11.77 \mathrm{~mm}$ for men and $11.64 \mathrm{~mm}$ for women as previously described [10, 11].

Using the center of the pupil as a reference point at $(0,0)$, $2 \mathrm{~mm}$ intervals from the midpupillary line were marked out medially for $8 \mathrm{~mm}$ and laterally for $10 \mathrm{~mm}$. This created 10 reference points for each eye, including the center of the pupil. From each of these points, a vertical line was drawn ending at the margin of the upper eyelid. The $x$ and $y$ coordinates of these points were recorded, with the $x$ coordinate signifying the horizontal distance from pupil center and the $y$ coordinate representing the vertical height. The $y$ measurement corresponding to $x=0$ represents the MRD1. This process (Fig. 1) was carried out for both preoperative and post-operative photographs in all eyes.

\section{Statistical analysis}

Comparison of pre-operative and post-operative measurements were made. The resultant difference in lid elevation at each of the 10 points on the lid were calculated. Comparisons of $y$ coordinate distances were made within and across groups with ANOVA analysis. Individual points were further compared across the two groups using the independent samples $t$-test. Analyses were performed with Statistical Package for the Social Sciences for Mac version 22.0 software (SPSS, IBM Inc., Armonk, New York, USA).

\section{Surgical technique}

\section{MMCR}

A standardized surgical method for MMCR was performed. Local anesthetic consisting of $2 \%$ lidocaine with 1:100,000 

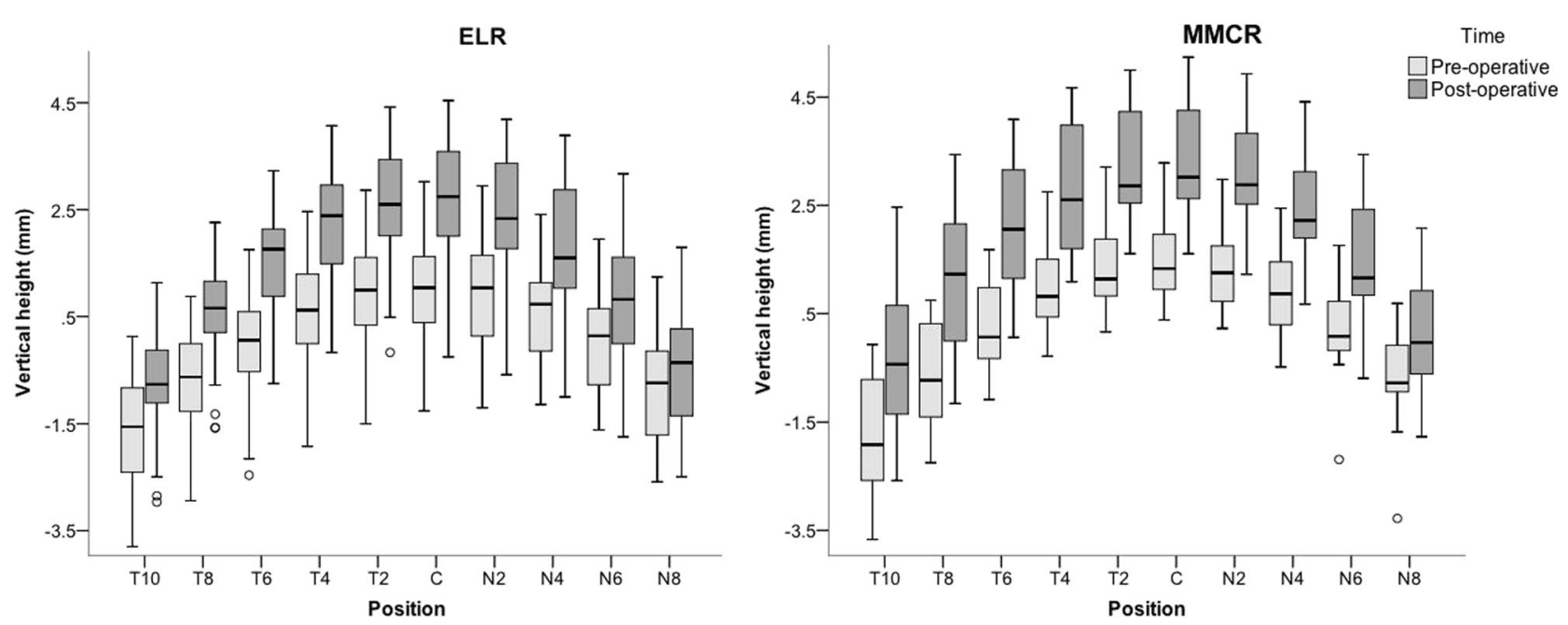

Fig. 2 Pre-operative and post-operative eyelid positions. ELR external levator resection, $M M C R$ Müllers muscle-conjunctival resection

epinephrine was injected into the upper eyelid. The eyelid was then everted. The desired length of tissue resection was marked on the conjunctiva using a caliper superior to the tarsus both laterally and medially. Two forceps were then used to elevate the conjunctiva-Müller's muscle composite flap. The ptosis clamp was then applied. Chromic gut suture was then driven under the clamp running in both directions with the ends externalized to skin. A \#15 Bard-Parker blade completed the resection.

\section{External levator resection}

Local anesthetic consisting of $2 \%$ lidocaine with 1:100,000 epinephrine was injected into the upper eyelid. The lid was approached through an eyelid crease incision. The tarsus was cleared at the superior border. Dissection on the posterior plane of the levator aponeurosis allowed identification of the levator posterior surface. A 6-0 novafil suture (Covidien, Dublin, Ireland) was passed through the tarsal plate designed to optimize the contour, and then through the posterior surface of the levator aponeurosis. The suture was adjusted to appropriate eyelid position and contour and then tied permanently; occasionally a second or third suture was placed to optimize eyelid contour. The skin incision was closed with 6-0 fast absorbing gut suture.

\section{Results}

A total of 60 eyes of 39 patients met criteria and were included in the analysis. The MMCR group contained 30 eyes (15 patients) and the ELR group consisted of 30 eyes (24 patients).
The overall main effect of position was significant for each group individually at both time points $(p<0.05$ in all cases) indicating there is variation in vertical height across the eyelid. The effect of time was also significant $(p<0.05$ in all cases) indicating that the vertical height of the eyelid changed with surgery at all positions for each group (Fig. 2).

When assessing individual point differences between groups, there was a significant difference $(p<0.05)$ in eyelid position at the temporal 2 and $4 \mathrm{~mm}$ points postoperatively only, with the ELR group being slightly higher at these positions post-operatively (Fig. 3). No other preoperative or post-operative comparisons were significant.

\section{Discussion}

In this study, we illustrated that both MMCR and ELR provide excellent eyelid elevation across the length of the eyelid. We did not find a difference in post-operative MRD1 between the MMCR and ELR groups. However, we did note a small but significant difference in eyelid height post-operatively between the MMCR and ELR groups at the 2 and $4 \mathrm{~mm}$ temporal positions, where the ELR eyelid was slightly higher on average, suggesting a difference in contour between the two groups.

Many groups have attempted to quantify eyelid contour. For example, Cruz and colleagues investigated the upper eyelid contour in patients with thyroid eye disease-related eyelid retraction. They used image processing software to place specific points along the lid, and then generated a closely fitted second-degree polynomial function. From this, information such as the degree of curvature, peak of contour, and areas of interest were obtained [12]. The authors used the 

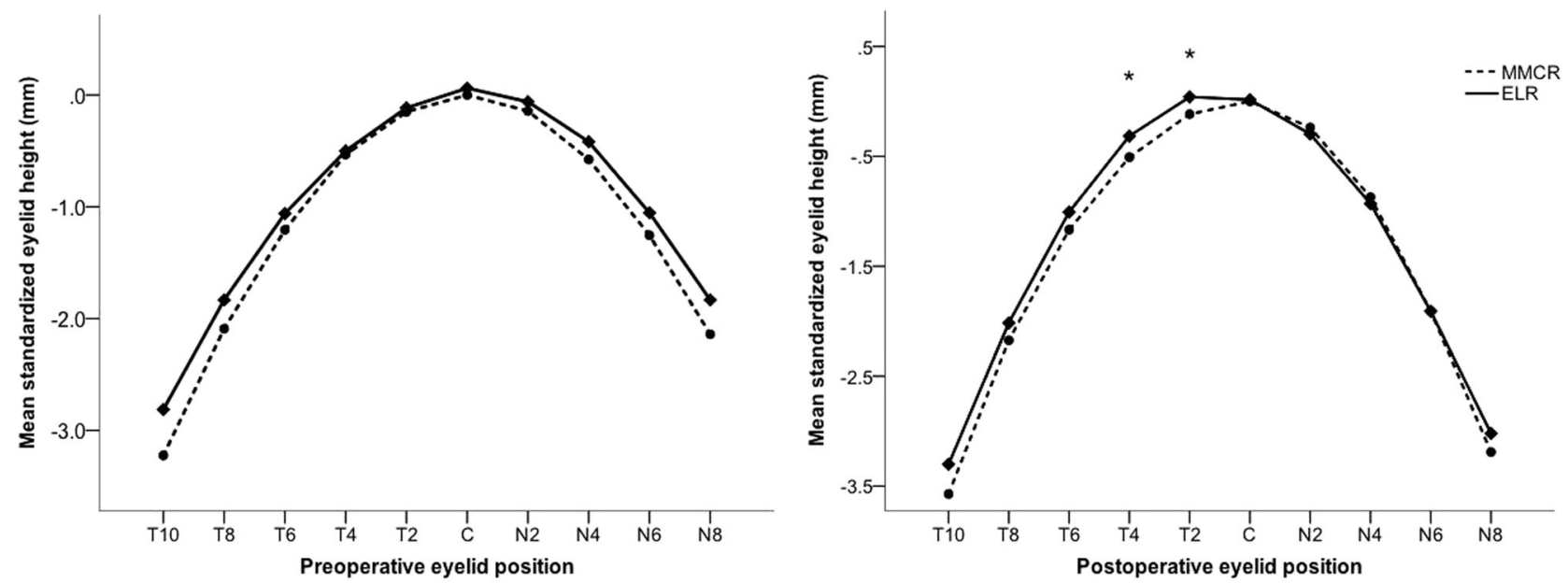

Fig. 3 Standardized baseline vertical eyelid height at 10 positions $(\mathrm{mm})$ across the eyelid in MMCR and ELR subjects for pre- and post-operative state. $(* p<0.05)$. ELR external levator resection, MMCR Müllers muscle-conjunctival resection

same approach in patients with congenital ptosis [12-14]. This graphical method is limited in that the eyelid in many physiologic and pathologic conditions may not follow a uniform parabolic function. Additionally, polynomial functions can be difficult to average across populations and to compare in a meaningful way between groups.

A more recent strategy described by the same group [12, $13,15,16]$ makes use of multiple radial mid pupil to upper eyelid margin distances (MPLDs) originating at the pupil center. This system directs the user to identify the center of the pupil, after which radial lines are drawn out automatically. The user then identifies the intersections of these lines with the eyelid margin. This has been used to quantify contour in patients with thyroid eye disease [17, 18] and after ptosis surgery [16]. Ahn et al. [19] used this method to assess eyelid contour after ELR, and were able to show quantitative improvement post-operatively as defined by an increase in MRD1 and MPLD angles.

One of the key difficulties of this approach relates to the non-uniform change in the eyelid parabola with elevation. As the eyelid is anchored medially and laterally, it does not elevate equally in all directions. Therefore, radial lines drawn from the center of the pupil will intersect with different positions along the eyelid margin pre- and post-operatively if there is a change in elevation following surgery. This creates a situation in which the pre-operative MPLD and post-operative MPLD are measuring different positions along the eyelid margin and therefore may not be compared directly. A similar challenge is presented in averaging across, or comparing, groups of eyelids.

The method described in this study defines stable positions on the eyelid meridian for measurements pre- and post-operatively. The measurements represent "multiple MRD1s" that are repeatable and can easily be compared clinically across time points and groups of patients. The main limitation of this method is the two dimensional nature of the analysis, a three dimensional assessment of the contour may be more accurate [20].

Despite the limitations in the method, we were able to demonstrate that both MMCR and ELR surgery are effective strategies for elevating the eyelid margin. The final postsurgical MRD1 was found to be equal for both approaches. These findings are supported by previous work [20].

We also noted a small lateralization of the peak with ELR surgery relative to MMCR. Lateralization of the eyelid peak has been reported previously after ELR surgery [21], and may have an anatomic basis [22]. In comparative studies [20], authors have used a subjective cosmetic outcome grading system to assess the outcome of MMCR surgery. This study did not include a qualitative assessment to correlate the aesthetic outcomes with lateralization, thus it is difficult to determine which eyelid contour is more normal or pleasing.

Some groups have suggested that eyelids may be slightly lateralized in normal [19] and younger subjects [23]. The corollary of this assessment is that post-operative lateralization might represent a return to a more "normal" contour, suggesting this effect would improve cosmesis. In this case, the MMCR effect of flattening the contour may be less desirable. It is not clear from our study or the available literature which contour would be considered superior in an objective sense, nor whether this relates to contour lateralization.

The technique described for measurement in this study represents further refinement of eyelid contour analysis, and although limited by its two dimensional nature, is a useful step in the evolution of these assessments. Using automated processes would significantly improve the clinical utility of such contour grading. With this system, we were able to demonstrate subtle post-operative differences in contour, 
with ELR patients maintaining a slightly higher eyelid position lateral to the center of the pupil.

\section{Summary}

\section{What was known before}

- At present there are no standardized, objective techniques to measure the eyelid contour The eyelid contour is flatter with the MMCR technique.

\section{What this study adds}

- A novel technique to objectively measure eyelid contour ELR and MMCR surgery achieve similar MRD1 measurements.

\section{Compliance with ethical standards}

Conflict of interest The authors declare that they have no conflict of interest.

\section{References}

1. Dailey RA, Wobig JL. Eyelid anatomy. J Dermatol Surg Oncol. 1992;18:1023-7.

2. Small RG, Sabates NR, Burrows D. The measurement and definition of ptosis. Ophthal Plast Reconstr Surg. 1989;5:171-5.

3. Elner VM, Hassan AS, Frueh BR. Graded full-thickness anterior blepharotomy for upper eyelid retraction. Trans Am Ophthalmol Soc. 2003;101:67-73-5.

4. Morris CL, Morris WR, Fleming JC. A histological analysis of the Müllerectomy: redefining its mechanism in ptosis repair. Plast Reconstr Surg. 2011;127:2333-41.

5. Carter KD, Frueh BR, Hessburg TP, Musch DC. Long-term efficacy of orbital decompression for compressive optic neuropathy of Graves' eye disease. Ophthalmology. 1991;98:1435-42.

6. Liu H, Shao Y, Zhang D. Surgical correction of blepharoptosis using a modified levator aponeurosis-Müller muscle complex reinsertion technique. J Craniofac Surg. 2014;25:226-30. http://www.ncbi.nlm.nih.gov/pubmed/24406583.

7. Melese M, Alemayehu W, Bejiga A, Adamu Y, Worku A. Modified grading system for upper eyelid trachomatous trichiasis. Ophthalmic Epidemiol. 2003;10:75-80.

8. Gower EW, West SK, Cassard SD, Munoz BE, Harding JC, Merbs SL. Definitions and standardization of a new grading scheme for eyelid contour abnormalities after trichiasis surgery. PLoS Negl Trop Dis. 2012;6:e1713.

9. Milbratz GH, Garcia DM, Guimares FC, Cruz AAV. Multiple radial mid pupil lid distances: a simple method for lid contour analysis. Ophthalmology. 2012;119:625-8.

10. Rüfer F, Schröder A, Erb C. White-to-white corneal diameter: normal values in healthy humans obtained with the Orbscan II topography system. Cornea. 2005;24:259-61.
11. Rootman DB, Karlin J, Moore G, Goldberg R. The role of tissue resection length in the determination of post-operative eyelid position for Muller's muscle-conjunctival resection surgery. Orbit. 2015;34:92-8. http://www.tandfonline.com/doi/full/10.3109/ 01676830.2014 .999096$.

12. Cruz AAV, Coelho RP, Baccega A, Lucchezi MC, Souza ADA, Ruiz EES. Digital image processing measurement of the upper eyelid contour in graves disease and congenital blepharoptosis. Ophthalmology. 1998;105:913-8.

13. Cruz AA, Lucchezi MC. Quantification of palpebral fissure shape in severe congenital blepharoptosis. Ophthal Plast Reconstr Surg. 1999;15:232-5.

14. Simon GJB, Lee S, Schwarcz RM, McCann JD, Goldberg RA. External levator advancement vs Müller's muscle-conjunctival resection for correction of upper eyelid involutional ptosis. Am J Ophthalmol. 2005;140:426-32.

15. Cruz AA, Akaishi PM, Coelho RP. Quantitative comparison between upper eyelid retraction induced voluntarily and by Graves orbitopathy. Ophthal Plast Reconstr Surg. 2003;19:212-5. http://www.ncbi.nlm.nih.gov/pubmed/12918557.

16. Cruz AAV, Akaishi PMS, Mendonça AKTS, Bernadini F, Devoto M, Garcia DM. Supramaximal levator resection for unilateral congenital ptosis. Ophthal Plast Reconstr Surg. 2014;30:366-71. http://content.wkhealth.com/linkback/openurl?sid=WKPTLP:la ndingpage $\& a n=00002341-201409000-00002$.

17. Ribeiro SFT, Milbratz GH, Garcia DM, Devoto M, Guilherme Neto H, Mörschbächer R, et al. Pre- and postoperative quantitative analysis of contour abnormalities in Graves upper eyelid retraction. Ophthal Plast Reconstr Surg. 2012;28:429-33. http://www. ncbi.nlm.nih.gov/pubmed/23138202.

18. Ribeiro SFT, Milbratz GH, Garcia DM, Fernandes VL, RochaSousa A, Falcão-Reis FM, et al. Lateral and medial upper eyelid contour abnormalities in Graves orbitopathy. Ophthalmic Plast Reconstr Surg. 2013;29:40-3. http://content.wkhealth.com/linkba ck/openurl?sid $=$ WKPTLP:landingpage $\&$ an $=00002341$ 201301000-00010\%5Cnpapers2://publication/doi/10.1097/IOP. 0b013e3182747537.

19. Ahn S, Lee H, Lee J, Park J, Park M, Baek S. Analysis of surgical outcome after levator advancement by assessing changes in eyelid contour. J Craniofac Surg. 2016;27:1147-50. http://content.wkhea lth.com/linkback/openurl?sid=WKPTLP:landingpage $\&$ an $=00001$ 665-201607000-00009.

20. Malbouisson JM, Baccega A, Cruz AA. The geometrical basis of the eyelid contour. Ophthal Plast Reconstr Surg. 2000;16:427-31. http://www.ncbi.nlm.nih.gov/pubmed/11106186.

21. Flynn TH, Rose GE, Shah-Desai SD. Digital image analysis to characterize the upper lid marginal peak after levator aponeurosis repair. Ophthal Plast Reconstr Surg. 2011;27:12-4. http://www. ncbi.nlm.nih.gov/pubmed/20940664.

22. Kakizaki H, Zako M, Ide A, Mito H, Nakano T, Iwaki M. Causes of undercorrection of medial palpebral fissures in blepharoptosis surgery. Ophthalmic Plast Reconstr Surg. 2004;20:198-201. http://content.wkhealth.com/linkback/openurl?sid=WKPTLP:la ndingpage $\&$ an $=00002341-200405000-00004$.

23. Lee H, Lee JS, Chang M, Park M, Baek S. Analysis of lid contour change with aging in Asians by measuring mid pupil lid distance. Plast Reconstr Surg. 2014;134:521e-9e. http://content.wkhealth. com/linkback/openurl?sid $=$ WKPTLP:landingpage $\& a n=00006534-$ 201410000-00008. 